\title{
A tennis field test to objectively measure the hitting accuracy based on an Excel spreadsheet: Practical guidelines and applications
}

Gabriel Delgado-García ${ }^{1,2 *}$, Jos Vanrenterghem ${ }^{3}$, Javier Courel-Ibáñez ${ }^{4}$ Emilio J. RuizMalagón ${ }^{1,2}$, Santiago Ruiz-Alias ${ }^{1,2}$ and Víctor Manuel Soto-Hermoso ${ }^{1,2}$

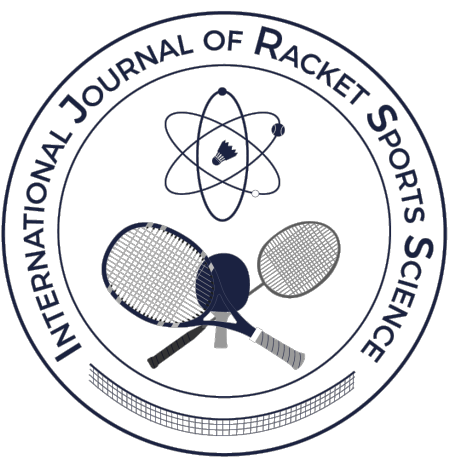

' Department of Physical Education and Sports, Faculty of Physical Activity and Sports Sciences, University of Granada, Spain.

${ }^{2}$ Sport and Health Research Institute (iMUDS), University of Granada, Spain.

${ }^{3}$ Department of Rehabilitation Sciences, Faculty of Kinesiology and Rehabilitation Sciences, KU Leuven, University of Leuven, Belgium.

${ }^{4}$ Department of Physical Activity and Sport, Faculty of Sport Sciences, University of Murcia, San Javier, Murcia, Spain.

\begin{abstract}
Stroke accuracy is highly related with tennis performance and has traditionally been quantified using general areas of scoring. Hence there is a need to develop methods that allow accuracy to be measured with higher resolution. The aim of the work is to develop a field test and an Excel spreadsheet associated that allows to evaluate the accuracy of the strokes with a resolution up to centimetres and to study how shots landings are distributed. The test consists of 4 series of 20 groundstrokes performed in the down the line or cross-court direction (this is modifiable). The 2D coordinates of bounce of the ball is recorded with a camera, digitalized using a specialized software and introduced in the Excel spreadsheet. Then it computes a series of parameters that describe the $95 \%$ confidence ellipse of the shot landing on the court. A real example of the test outcomes of two advanced players performing forehands and backhands down the line- is shown. Consistent with previous literature both players obtained a better accuracy in the mediolateral direction than in the longitudinal direction and ellipses were oriented almost parallel to the sideline (ellipse tilts were below 12 degrees in all cases). Ellipse area was considerably greater for the backhand than for the forehand in player two $\left(38.8 \mathrm{vs} .55 .5 \mathrm{~m}^{2}\right)$ but not in player one $\left(51.5 \mathrm{vs} .50 .8 \mathrm{~m}^{2}\right)$. Finally, the centre location of the ellipse in the longitudinal axis was positive in all cases (near $200 \mathrm{~cm}$ ) which suggest that both players preferred to make short shots rather than send the ball out of the limits of the baseline. We conclude that this methodology can be used by researchers that want to assess accuracy with high resolution and by coaches that want to evaluate -with high sensibility- the player progression after a training program.
\end{abstract}

Keywords: Tennis, Stroke Precision, Testing, Racket Sports, Software

Correspondence author: Gabriel Delgado-García

E-mail: gabrieldg@ugr.es

\footnotetext{
Cite this article as:

Delgado-García, G., Vanrenterghem, J., Courel-Ibáñez, J., Ruiz-Malagón, E., Ruiz-Alias, S., \& Soto-Hermoso, V. (2019). A tennis field test to objectively measure the hitting accuracy based on an Excel spreadsheet:

Practical guidelines and applications. International Journal of Racket Sports Science, 1(2), 24-36. 


\section{Introduction}

Tennis performance depends, among other factors, on tennis hitting accuracy (Landlinger, Stöggl, Lindinger, Wagner, \& Müller 2012). There is an extended bibliography that shows specific tennis field tests for measuring it, but most of those tests establish a categorical system of punctuations based on landing areas (Baiget, Fernández-Fernández, Iglesias, Vallejo, \& Rodríguez, 2014; Davey, Thorpe, \& Williams, 2003; Smekal et al., 2000; Strecker, Foster, \& Pascoe, 2011). Generally, in most of those tests a ball that hits the corner of the court has a higher score than a ball that lands in the middle. They are limited and for example cannot differentiate between a ball that touches the line and a ball that lands out by a minimal distance. There are a few specific tennis field protocols that have been used to evaluate the accuracy in strokes with centimetre accuracy (Delgado-García et al., 2019; Landlinger et al., 2012; Vergauwen, Spaepen, Lefevre \& Hespel, 1998; Yamamoto, Shinya, \& Kudo, 2018). They have been used to: compare the accuracy of strokes based on the direction of the strokes and level of the player (Landlinger et al., 2012); compare the accuracy depending on the type of stroke (DelgadoGarcía et al., 2019; Landlinger et al., 2012); analyse the effect of fatigue on accuracy (Vergauwen, Lieven, Brouns, Fred, \& Hespel, 1998) or relate the accuracy with cognitive aspects (Yamamoto et al., 2018). Despite these tests, little information is available on how to evaluate accuracy in the particular case of tennis. There is still a need to develop tools and methodologies that allow trainers and scientists to assess accuracy with sufficiently high resolution.

The development of new technologies applied to sport is still on a rise. Although there are affordable devices to measure the speed of hitting (speed radars and inertial sensors), there are not many instruments to measure the accuracy of ball placement. There are some expensive systems that provide tennis match analytics such as the Hawk-eye (Hawk-Eye Innovations, 2016) or the smart tennis courts mounted by the company PlaySight (PlaySight Interactive, Ltd., Kokhav Ya'ir, Israel). The Hawk-eye is used during high level tournaments and the data that provide have been used in different research works (Reid, Morgan, \& Whiteside, 2016; Wei, Lucey, Morgan, \& Sridharan, 2013). Other solutions are shown in the scientific bibliography such as the proposed by Messelodi, Modena, Ropele, Marcon \& Sgrò (2019) or Wawrzyniak \& Kowalski (2016). However, there are hardly any low-cost and ease of use technologies that have a similar purpose to these systems. In this regard, we could mention the Swing Vision tennis app for iOS (Mangolytics Inc., 2019), based on artificial intelligence. Another tool a little more expensive is Mojjo, that uses two simple mobile phone cameras (Mojjo, 2019, Paris, France). It is also interesting to mention the "In / Out" device, a double camera that is placed on the net and that performs a mapping of the location of the ball landing on the court (according to the manufacturers it has millimetre accuracy). Furthermore, there is specialized software that allows digitisation the ball landing location (e.g., Kinovea, Tracker, SkillSpector, Check2D) and therefore study its accuracy. Relevant ball trajectory data can be obtained with this software, but it remains difficult to extract parameters related to accuracy. Excel is a software that many scientists and coaches use on a daily basis and that allows semi- or even fully-automated mathematical calculations for sports analysis. For example, Chavda et al. (2018) designed an Excel spreadsheet that serves to analyse force platform data. Another Excel application allows running automated algorithms for biomechanical data analysis such as data filtering, interpolation, differentiation, integration, etc. (Biomechanics Toolbar, Vanrenterghem, 2016). In a recent study (Delgado-García et al., 2019) the distribution of groundstrokes was analysed using confidence ellipses created with a statistical package for Excel which allows to fit a scatter plot with a bivariate normal distribution (Zaiontz, 2015). This kind of distribution has been used in other sport science research, such as in posturography assessments (Schubert \& Kirchner, 2014).

Therefore, the objective of this work was to propose a field hitting test based on previous literature and describe an accompanying Excel tool, which allows the calculation of confidence ellipses of a tennis player for the forehand and backhand stroke. This tool can be 
used by coaches and scientists who want to objectively and with centimetre resolution assess the accuracy of tennis players. We will show an exemplary usage of the tool with two advanced tennis players.

\section{Methods}

\section{Sample}

Two experienced tennis players volunteered to complete a hitting field-test including 80 strokes each $(n=160)$. The two players had more than 20 years of playing experience and an international level number of three which correspond with advanced players (ITF, 2019). For descriptive purposes their body composition was measured with bioimpedance (Inbody 230, Biospace, Korea). Player 1 was 49 years old (mass $=80 \mathrm{~kg}$; skeletal muscle mass $=34.8 \mathrm{~kg}$, body fat percentage $=24 \%$ and IMC $=29.2$ ). Player 2 was 33 years old (mass $=74.8 \mathrm{~kg}$; skeletal muscle mass $=36.9 \mathrm{~kg}$, body fat percentage $=13.7 \%$ and IMC $=$ 23.3). Both participants were right-handed, used a onehanded backhand and didn't report any musculoskeletal injury that would limit their stroke or shifting technique or the use of drugs due to serious illness. They were informed of the benefits and risks of the investigation prior to signing an institutionally approved informed consent according to ethical principles for medical research involving human subjects as defined by the Declaration of Helsinki.

\section{Procedures}

\section{Set-up of the field tennis test}

The proposed hitting test consists of 4 series of 20 groundstrokes each (80 strokes in total). The evaluator can select four different types of test explained in Table 1. In the example shown in this manuscript the players were told to hit forehand and backhands down the line (test type 1) and to find the centre of the target while maintaining a similar pace to competition pace. The sheet can be used for other types of strokes such as volleys, approach shots, backspin strokes, lobs, etc. The only premise that must be met is that the shots must be made to the right half and left half of the opposite track alternately. If the test measures the accuracy in terms of proximity to the objective but also considering the number of errors, the initial information provided to the participant must specify it, since this factor can condition the player to take a greater or lesser risk with their strokes and will probably change the test results. For the present study the target was located inside the court at a distance of $1 \mathrm{~m}$ from the sideline and the baseline (Figure 2) but the evaluator can select other locations. It is recommended to perform a warm-up of about $8 \mathrm{~min}$ (3 min of running and mobility exercises, 3 min of rally with another player and 2 min performing a series of the test). To ensure that fatigue does not affect the results of each series participants should be given 3-5 min rest between them so as to allow their heart rate to return to resting levels or within 10 beats/min of resting levels (Lyons, Al-Nakeeb, Hankey, \& Nevill, 2013) which can be assessed with the use of a pulsometer. We recommend the use of a device that has been validated in the scientific bibliography, such as the polar RS400 which has been found valid and reliable during both physical activity and exercise training (Engström et al., 2012).

To ensure that all players perform the test in the same conditions of pace and speed of the ball, a ball throwing machine can be used. For example in the study of Lyons et al. (2013) they use a Tennis Tutor Plus (Sports Tutor, USA) and the speed of release was configured around $70 \mathrm{~km} / \mathrm{h}$ with a little of topspin so the ball travels over the net at a height of approximately $1.5 \mathrm{~m}$ and lands within approximately 2 $\mathrm{m}$ of the baseline. For the present study a Lobster Grand Slam IV in the predetermined configuration mode called "Two lines" was used. The throwing rate was of 20 throws per minute and the release speed of $70 \mathrm{~km} / \mathrm{h}$. The shot distance to the centre of the court was configured as medium, and the distance to the baseline was configured as row A. Finally, the spin level 1 was selected (from 3 positive levels, being 0 the flat shot and 3 the highest topspin allowed by the machine). 
Table 1.

Possible test settings and order of the strokes depending on the dominant hand of the player

\begin{tabular}{|c|c|c|c|c|c|}
\hline \multirow{3}{*}{ Configuration } & \multirow{3}{*}{ Description } & \multicolumn{4}{|c|}{ Order of the strokes } \\
\hline & & \multicolumn{2}{|c|}{ Right-handed players } & \multicolumn{2}{|c|}{ Left-handed players } \\
\hline & & 1st stroke & 2nd stroke & 1st stroke & 2nd stroke \\
\hline Test 1 & $\begin{array}{l}\text { Forehand and backhand alternately } \\
\text { in the down the line direction }\end{array}$ & $\begin{array}{l}\text { Down the } \\
\text { line forehand }\end{array}$ & $\begin{array}{l}\text { Down the line } \\
\text { backhand }\end{array}$ & $\begin{array}{l}\text { Down the line } \\
\text { backhand }\end{array}$ & $\begin{array}{l}\text { Down the line } \\
\text { forehand }\end{array}$ \\
\hline Test 2 & $\begin{array}{l}\text { Forehand and backhand alternately } \\
\text { in the cross-court direction }\end{array}$ & $\begin{array}{l}\text { Cross-court } \\
\text { backhand }\end{array}$ & $\begin{array}{l}\text { Cross-court } \\
\text { forehand }\end{array}$ & $\begin{array}{l}\text { Cross-court } \\
\text { forehand }\end{array}$ & $\begin{array}{l}\text { Cross-court } \\
\text { backhand }\end{array}$ \\
\hline Test 3 & $\begin{array}{l}\text { Forehand down the line and } \\
\text { forehand cross-court alternately }\end{array}$ & $\begin{array}{l}\text { Down the } \\
\text { line forehand }\end{array}$ & $\begin{array}{l}\text { Cross-court } \\
\text { forehand }\end{array}$ & $\begin{array}{l}\text { Cross-court } \\
\text { forehand }\end{array}$ & $\begin{array}{l}\text { Down the line } \\
\text { forehand }\end{array}$ \\
\hline Test 4 & $\begin{array}{l}\text { Backhand cross-court and backhand } \\
\text { down the line alternately }\end{array}$ & $\begin{array}{l}\text { Cross-court } \\
\text { backhand }\end{array}$ & $\begin{array}{l}\text { Down the line } \\
\text { backhand }\end{array}$ & $\begin{array}{l}\text { Down the line } \\
\text { backhand }\end{array}$ & $\begin{array}{l}\text { Cross-court } \\
\text { backhand }\end{array}$ \\
\hline
\end{tabular}

To assign a value to these numbers in a unit understandable to the rest of the members of the scientific community, a small study was conducted in order to determine the estimated location of the bounce of the ball, the spin of the ball in revolution per minute (revs/min) and the height the ball pass over the net, in the selected mode of the throwing machine ("Two lines"). In this studio, the position of the bounces of the balls sent by the machine was recorded using a $60 \mathrm{~Hz}$ rear camera (iPhone 6) and digitized in the Kinovea software (a total of 80 balls were digitized: 40 on the right side and 40 to the left side). The coordinates obtained were entered on the Excel spreadsheet that will be explained in this manuscript and the parameters of the confidence ellipses shown in Figure 1 were obtained. The spin of the ball was determined using coloured balls in such a way that it could be visualize the number of turns the ball takes (14 throws were analysed). The ball's departure from the machine was recorded with a camera sampling at $1000 \mathrm{~Hz}$ (Sony RX100 IV) and the time it took for the ball to make two turns was counted in Kinovea moving frame by frame. Subsequently, the speed of rotation of the ball in revs/min was calculated. The height of the flight of the ball over the net was calculated by placing the machine at a distance of $1188.5 \mathrm{~cm}$ (the distance from the baseline to the net) respect to a vertical wall of $5 \mathrm{~m}$ height. The machine shot in such a way that all the balls entered into a rectangle painted on the wall of $336 \mathrm{~cm}$ high by $182.4 \mathrm{~cm}$ wide and the impact of the ball on it was recorded at $60 \mathrm{~Hz}$ (iPhone 6s) and digitized in Kinovea (a total of 40 impacts were digitized). The results of these tests are shown in Figure 1. It is important to remark that if the user of the spreadsheet would like to perform the test type 3 or 4 (Table 1) the machine should be configured in such a way that all the throws are directed to one side of the court (the player only perform forehands or backhands strokes in this two test).

Alternatively, the ball can be thrown by an expert coach (Delgado-García, Vanrenterghem, MuñozGarcía, Molina-Molina, \& Soto-Hermoso, 2018), using a metronome and trying to throw all the balls in the same way. It is recommended to throw the ball at a rate of one throw every three seconds (DelgadoGarcía et al., 2019; Lyons et al., 2013). 


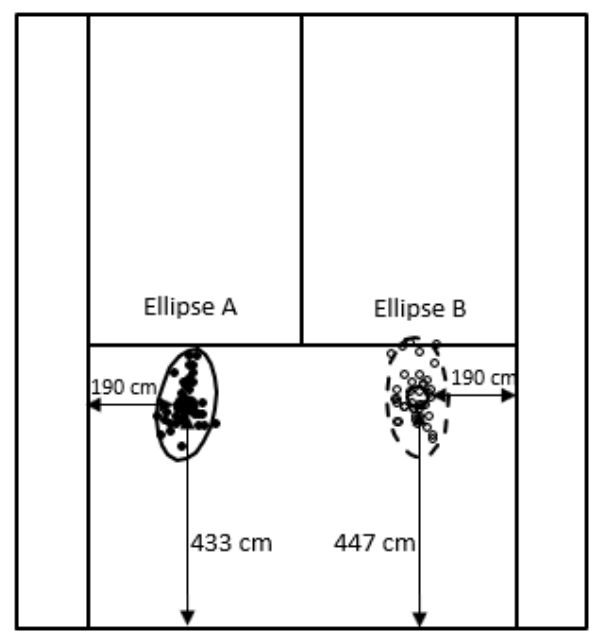

Figure 1. Location of the bounces from the throwing machine Lobster Grand Slam IV in the preconfigured mode "Two Lines". The figure shows the distance of the centre of the ellipse to the sideline and to the baseline. The ellipse A has a major axis of $108 \mathrm{~cm}$ a minor axis of $55 \mathrm{~cm}$ and an area of $1.9 \mathrm{~m}^{2}$. The ellipse $B$ has a major axis of $118 \mathrm{~cm}$, a minor axis of $59 \mathrm{~cm}$ and an area of $2.2 \mathrm{~m}^{2}$. The spin of the ball was of 855 $\pm 55 \mathrm{rpm}$ and the height of the ball over the net was of $246 \pm 9 \mathrm{~cm}$.

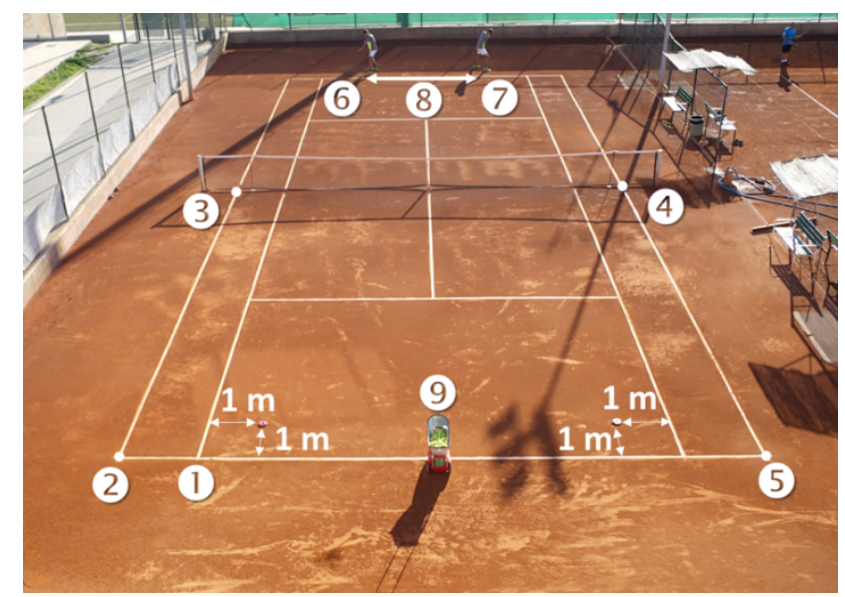

Figure 2. Set-up of the tennis test (view from the camera that records the bounce of the ball) used in this research work. Meaning of the numbers: origin of coordinates (1); corner of the perspective grid in Kinovea (2 to 5); approximate location of 1 st stroke (6); approximate location of 2nd stroke (7); displacement of the player (8); ball throwing machine (9).
The test is recorded with a camera placed behind and above the court, so that the full court is seen (including both backgrounds).

Figure 2 shows a camera setting that could be used. It is recommended that: I) The camera has a symmetrical view of the court (to reduce the perspective error); II) the camera records at a minimum sampling frequency of $60 \mathrm{~Hz}$ (to capture the impact of the ball on the court); III) the bottom of the court is recorded, with a margin of at least $4 \mathrm{~m}$ from the baseline (so as not to lose impacts that go too long).

After performing the 80 strokes, the bounces of the ball can be digitized using specific software. The coordinate origin must be selected in the corner of the backhand part of the court (for a right-handed player), on the side where the targets are located. Within a Windows environment Kinovea software is recommended (www.kinovea.org). A valid alternative for Mac environment is the Tracker Video Analysis and Modelling Tool (https://physlets.org/tracker/). In order to reduce the measurement error, it is recommended prior to performing the test to place a series of balls at known points on the court (see Figure 3) and calculate a regression line, which predicts the real bounce of the ball based on the digitized 2D coordinates (DelgadoGarcía et al ., 2019), or apply the 2D DLT calibration algorithm (Vergauwen et al., 1998). Another option is to use specific software, such as SkillSpector or Check2D (Dunn et al., 2012). Once the coordinates of the ball bounces are obtained (expressed in $\mathrm{cm}$ ), these coordinates are entered in the Excel spreadsheet. This then computes the $95 \%$ confidence ellipse, which covers with a $95 \%$ of probability the true population mean (Schubert \& Kirchner, 2014), for the forehand and the backhand stroke, in addition to a series of parameters related to the way in which the shots are distributed in the space: the length of both axes of the ellipse, the tilt of the ellipse, its eccentricity, the area and the location of the centre of the ellipse on the $\mathrm{x}$-axis and on the y-axis. Supplemental theorical information about the confidence ellipses and about parameters described can be found in the works of Delgado-García et al. 
(2019), Schubert \& Kirchner (2014), Shinya et al. (2017) or Yamamoto et al. (2018). To better understand the maths underlying the calculation of confidence ellipses, it is recommended to consult the "Real Statistic Using Excel Package" page (Zaiont, 2016), which is where Excel formulas have been extracted from. The present article will focus primarily on explaining how the Excel sheet is used and how to interpret the results, rather than on the mathematical and/or statistical underpinnings.

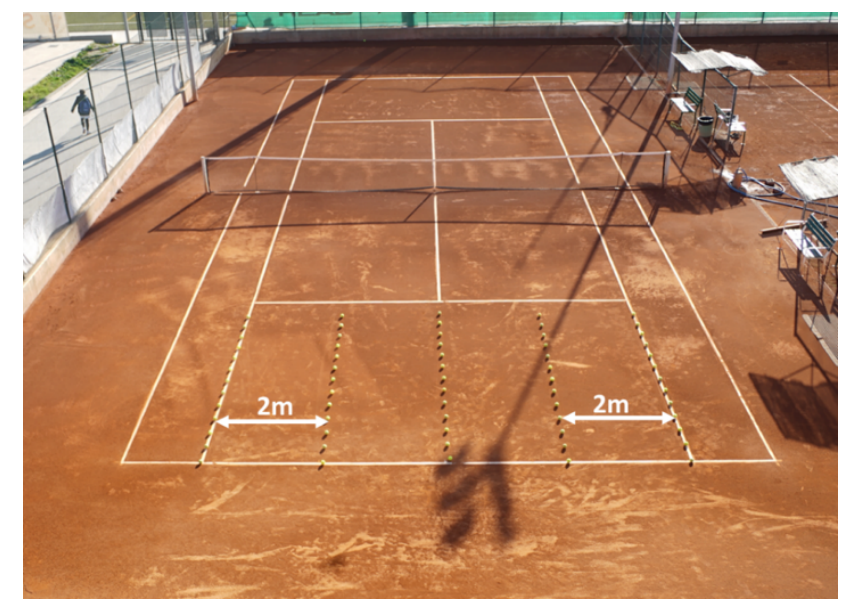

Figure 3. Example of a set of balls located at known positions and used to compute the regression line that allows to predict the real bounce of the ball based on the digitised bounce. The balls in each column have a separation between them of $40 \mathrm{~cm}$.

\section{How does the Excel book work?}

The Excel spreadsheet can be downloaded from here. This link also includes a videotutorial of the use of this Excel sheet and some real examples. Instructions appear in the first Excel spreadsheet ("Instructions"). It explains how the 2D-coordinates of the bounce of the ball are obtained using Kinovea software. In the spreadsheet "Accuracy analysis" only grey shaded cells should be modified. The user must indicate the dominance of the tennis player in cell E5 (with the exact words in uppercase "RIGHT" or "LEFT"), the distance of the centre of the target respect to the sideline (cell E6) and the baseline (cell E7) and the type of test performed (there are four possible tests, explained in Table 1). After that the user must paste the 2D coordinates of the ball bounces (in centimetres) obtained from Kinovea in range E10:F89. Once the 80 hits of the test have been pasted (it is necessary to fill in the 80 rows so that the mathematical calculations are correct), the following data are automatically produced:

- The length of the $95 \%$ confidence ellipse axes (cells V15 and V16 and AB15 and AB16).

- The angle that forms the long axis of the $95 \%$ confidence ellipse with the baseline (V17 and V18 and $\mathrm{AB} 17$ and $\mathrm{AB} 18$ ) and with the sideline (an angle of 0 degrees indicates that said axis is completely parallel to the sideline) in cells V19 and AB19. Since this information is repetitive, for the interpretation of the results only the angle with respect to the sideline (tilt) will be used. If the ellipse is oriented towards the inside of the court the tilt will be positive (direction changed for the ellipses of the forehand and backhand strokes).

- $\quad$ Eccentricity of the $95 \%$ confidence ellipse in cells V20 and AB20. This value ranges from 0 to 1 . A value of 0 corresponds to a circle. The closer to one this value is, the more eccentric the ellipse is (more oval shape).

The $95 \%$ confidence ellipse area in cells V21 and AB21. It gives information about global accuracy. A higher area means a less accurate stroke.

The distance from the centre of the ellipse to the centre of the target on the $\mathrm{x}$-axis and on the $\mathrm{y}$ axis (cells V22 and V23 and AB22 and AB23). It is generated with the means of the coordinates of the bounces of the balls on the $\mathrm{x}$ and y axes (cells G94, G96, G107 and G109) and with the distance of the target to the sideline (cell E6) and to the baseline (cell E7). A negative value indicates that the centre of the ellipse is between the centre of the target and the sideline and a positive value indicates that it is closer to the centre of the court. Those values give an idea of the trend of the shots.

Columns G contain the errors: the shot that bounce out of the limits of the singles tennis courts ("out"), the ball that bounce out of the limits of the camera field of view ("out of view") and the balls that does not pass over the net ("net"). The sum of those three values is the total of errors of the player. The balls that go out from the singles tennis court are considered for plotting the $95 \%$ confidence ellipse. 
Columns H:I and L:S are used to calculate the different parameters of the ellipse. As mentioned, all these calculations are well explained on the Zaiontz (2016) website. The confidence ellipse is generated with the covariance matrix of the $\mathrm{x}$ and $\mathrm{y}$ coordinates of the ball bounces of each of the strokes (shown in cell ranges R15:S16 and R31:S32). The covariances matrices allow to compute the two higher eigenvalues, assuming that the trace of the covariance matrix and the product of the eigenvalues is equal to the determinant of the matrix. These equations (two for each stroke) are solved with parameters in cells P23:S23 and P39:S39 and eigenvalues are shown in cells R25 and R26 (for 1st stroke) and R41 and R42 (for 2nd stroke). The remaining formulas are shown in table 2 .

Table 2.

Excel formulas used to compute confidence ellipse parameters

\begin{tabular}{|c|c|c|}
\hline Parameter & $1^{\text {st }}$ Stroke & $2^{\text {nd }}$ Stroke \\
\hline a-axis & $=\mathrm{SQRT}(\mathrm{MAX}(\mathrm{R} 25: \mathrm{R} 26)) * \mathrm{SQRT}(\mathrm{S} 27)$ & $=\mathrm{SQRT}(\mathrm{MAX}(\mathrm{R} 41: \mathrm{R} 42)) * \mathrm{SQRT}(\mathrm{S} 43)$ \\
\hline b-axis & $=\operatorname{SQRT}(\mathrm{MIN}(\mathrm{R} 25: \mathrm{R} 26)) * \mathrm{SQRT}(\mathrm{S} 27)$ & $=\operatorname{SQRT}(\mathrm{MIN}(\mathrm{R} 41: \mathrm{R} 42)) * \mathrm{SQRT}(\mathrm{S} 43)$ \\
\hline$\theta(\mathrm{rad})$ & $\begin{array}{l}=(\operatorname{ATAN} 2(\mathrm{~S} 15 ; \mathrm{MIN}(\mathrm{R} 25: \mathrm{R} 26)- \\
\operatorname{MAX}(\mathrm{R} 15 ; \mathrm{S} 16)))^{*}-1\end{array}$ & $\begin{array}{l}=(\operatorname{ATAN} 2(\mathrm{~S} 31 ; \mathrm{MIN}(\mathrm{R} 41: \mathrm{R} 42)- \\
\operatorname{MAX}(\mathrm{R} 31 ; \mathrm{S} 32)))^{*}-1\end{array}$ \\
\hline$\theta(\mathrm{deg})$ & $=$ DEGREES $(\mathrm{V} 17)$ & $=$ DEGREES $(\mathrm{AB} 17)$ \\
\hline Tilt (deg) & $=90-\mathrm{V} 18$ & $=\mathrm{AB} 18-90$ \\
\hline Eccentricity & $=(1-(\mathrm{V} 16 / \mathrm{V} 15) \wedge 2) \wedge 0.5$ & $=(1-(\mathrm{AB} 16 / \mathrm{AB} 15) \wedge 2) \wedge 0.5$ \\
\hline Area $\left(\mathrm{m}^{2}\right)$ & $=\left(\mathrm{V} 15^{*} \mathrm{~V} 16^{*} \mathrm{PI}()\right) / 10000$ & $=\left(\mathrm{AB} 15^{*} \mathrm{AB} 16^{*} \mathrm{PI}()\right) / 10000$ \\
\hline CE-x (cm) & $=\mathrm{G} 94+(100-\mathrm{E} 6)$ & $=(100-\mathrm{E} 6)-\mathrm{G} 107$ \\
\hline CE-y (cm) & $=\mathrm{G} 96+(100-\mathrm{E} 7)$ & $=(\mathrm{G} 109+100)-\mathrm{E} 7$ \\
\hline
\end{tabular}

a-axis: long axis of the confidence ellipse; b-axis: short axis of the confidence ellipse; CE-x: Confidence ellipse centre distance on the $\mathrm{x}$-axis to centre of the target; CE-y: Confidence ellipse centre distance on the $\mathrm{y}$-axis to centre of the target.

In addition to this data, two graphs are generated:

- $\quad$ Graph 1. Superposition of both confidence ellipses. This graph allows the user to compare visually the 1st stroke and 2nd stroke ellipses. It is also possible to see the centre of the ellipses relative to a common centre of coordinates. The backhand ellipse is rotated horizontally so as it can be compared with the forehand ellipse (the targets for both strokes are symmetrical).

- $\quad$ Graph 2. Shot distribution in the tennis court and confidence ellipses. It shows the disposition of the ellipse of each stroke on the tennis court (at real scale). Cells AC72 AND AC73 show the stroke to which each of the ellipses correspond (taking into account the lateral dominance of the player). In this graph it is also possible to visually check the distribution of the bounces and the centre of both ellipses relative to the centre of each target. The scatter plot of the landing of the strokes is generated with columns J and $\mathrm{K}$.

In the "Report for the tennis player" spreadsheet of the Excel workbook a report is displayed (the data is extracted from the Excel spreadsheet "Accuracy Analysis") that can be given to the player. The sheet "Interpreting the report" provides some reference values with which the results of the player performing the test can be compared. These values have been taken from the database of a previous research work (Delgado-García et al., 2019). In the "Court and ellipse coordinate" spreadsheet there are the $\mathrm{x}$ and $\mathrm{y}$ coordinates of the confidence ellipses and of the tennis court, information needed to create 
graphs 1 and 2 of the "Accuracy analysis" spreadsheet.

\section{Results}

Table 3 shows the parameters of the confidence ellipse of both players, for the forehand and backhand groundstrokes performed in the down the line direction. Figure 4 and 5 present this information in a visual way. The length of the major axis of player one for the forehand is greater than that of player two. In the backhand, player two has a longer major axis. The minor axis is longer in both player for the backhand than for the forehand. The angle formed by the ellipse with the sideline (ellipse tilt) is very similar in both cases. However, the backhand confidence ellipse has a greater inclination in player one. As for eccentricity, all ellipses have an oval shape, except the ellipse of the backhand of player one, which looks more like a circle. The forehand ellipse area of player two is small compared to the other three ellipses analysed. Only the centre of the ellipse on the x-axis of player one's backhand stroke was positive. The rest of the centres on the x-axis were negative, remaining between the $y$-axis and the sideline. On the $y$-axis this value was positive giving an idea of conservative behaviour on the part of both players (they preferred to perform short shots rather than risking to play beyond the baseline). Maybe if the player had been instructed that the out errors did not produce any kind of penalty and that the shots had to be adjusted as closely as possible to the centre of the target, they would have obtained values in this variable closer to zero. Regarding the number of errors, player two made seven more mistakes (11 vs. 18) with the backhand than player one.

Table 3.

Confidence ellipse parameters for forehand and backhand ellipses of both players analysed

\begin{tabular}{|c|c|c|c|c|}
\hline \multirow{2}{*}{ Parameter } & \multicolumn{2}{|c|}{ Player no. 1} & \multicolumn{2}{|c|}{ Player no. 2} \\
\hline & Forehand & Backhand & Forehand & Backhand \\
\hline a-axis $(\mathrm{cm})$ & 585 & 446 & 517 & 608 \\
\hline b-axis $(\mathrm{cm})$ & 280 & 363 & 238 & 291 \\
\hline Tilt (deg) & 6.5 & 11.7 & 2.8 & 0.8 \\
\hline Eccentricity & 0.88 & 0.58 & 0.89 & 0.88 \\
\hline Area $\left(m^{2}\right)$ & 51.5 & 50.8 & 38.8 & 55.5 \\
\hline CE-x (cm) & -11 & 45 & -40 & -34 \\
\hline CE-y (cm) & 171 & 238 & 191 & 225 \\
\hline Errors (n) & 16 & 11 & 16 & 18 \\
\hline Out shots (n) & 8 & 9 & 13 & 16 \\
\hline Out of camera view (n) & 8 & 2 & 1 & 1 \\
\hline Net errors (n) & 0 & 0 & 2 & 1 \\
\hline
\end{tabular}

a-axis: long axis of the confidence ellipse; b-axis: short axis of the confidence ellipse; CE-x: Confidence ellipse centre distance on the $\mathrm{x}$-axis to centre of coordinates; CE-y: Confidence ellipse centre distance on the y-axis to centre of coordinates. Out shots: Balls that bounce out of the limit of the singles tennis court. Out of camera view errors: balls that pass over the net but bounce outside the field of view of the camera. Net errors: balls that do not pass over the net. 

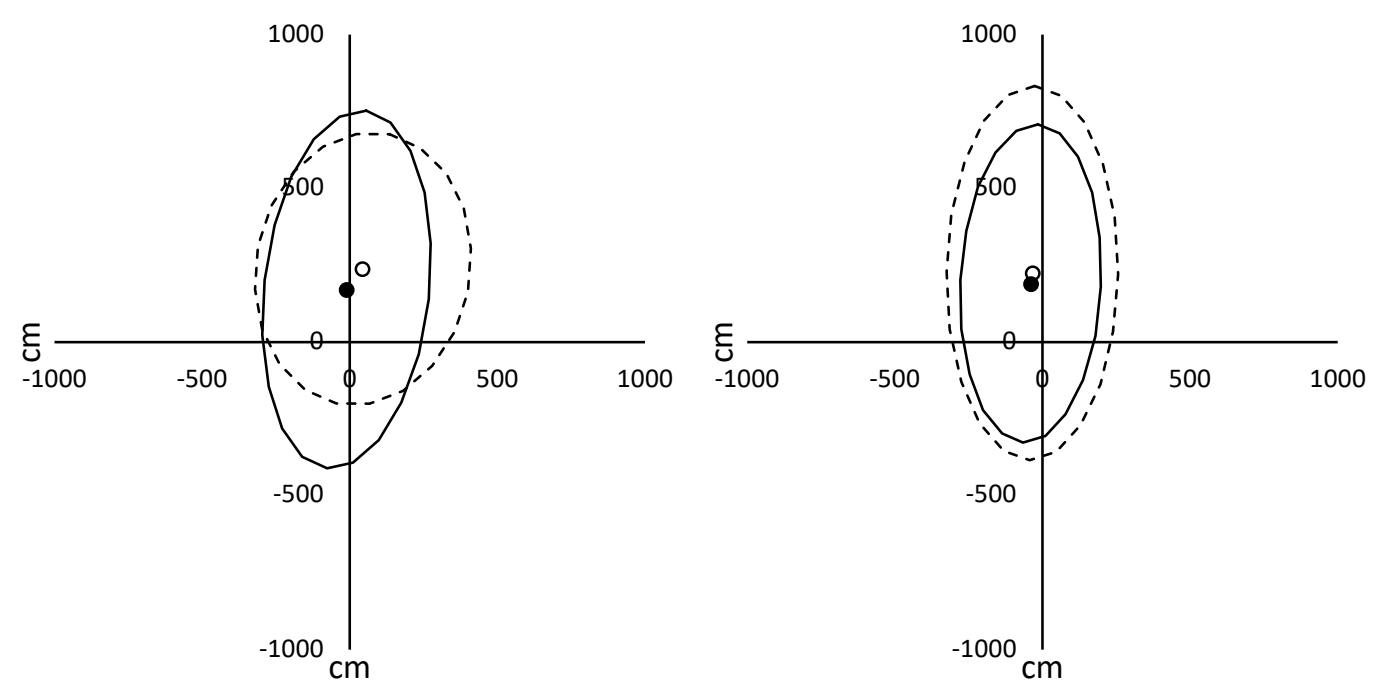

Figure 4. Confidence ellipses of player one (left) and player two (right) for the forehand (solid ellipse) and backhand (dashed ellipse) strokes.

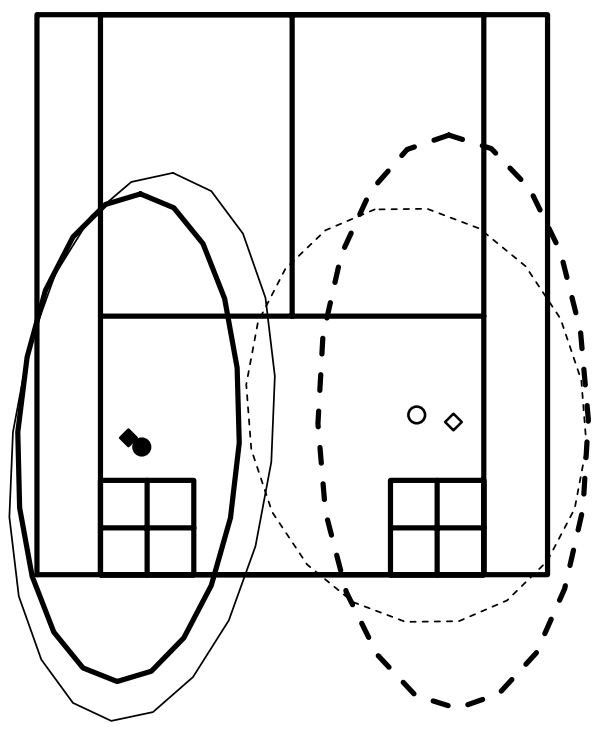

Figure 5. Confidence ellipses displayed relative to the tennis court. Forehand ellipses are in solid lines and backhand ellipses in dashed lines. Confidence ellipses of player two are marked in bold. The centres of the ellipses are indicated by circles (player one) or diamonds (player two).

\section{Discussion}

The test presented does not require expensive materials or a complicated assembly. It is only necessary to place a visible object in the centre of the targets and a camera at the bottom of the court recording the location of the bounces. It can be done by any coach or scientist and provides interesting information about the distribution of shots in space. It can be used in players of any level by modifying the distance and location of the target, the hitting speed and parameters related to the ball the player receives (frequency of the ball throws of the machine, speed and arrival effect, location of the court to which the 
ball bounces both in longitudinal and medio-lateral direction).

In the case of the present study both analysed players showed ellipses of similar characteristics. The area of the ellipse of player one's forehand was very similar to the ellipse area of his backhand. Player two did show a larger area with the backhand, which indicates that this stroke was less accurate. This is consistent with the data in the scientific literature (Delgado-García et al., 2019). Also, the major axes of the ellipses were oriented almost parallel to the sideline as in the mentioned manuscript of Delgado et al. (2019) which has been explained by the Calvin's Launch Window Hypothesis (1983). This theory suggests that the timing of the release of the projectile condition the spatial accuracy. In the particular case of tennis the racket reach high speeds which could difficult the "optimum window of release" (Delgado-García et al., 2019). This oval shape of the ball bounce distribution was more evident for the forehand strokes of both players. Based on this result both players could be advised to focus more on improving longitudinal accuracy rather than medio-lateral accuracy on the forehand. The same goes for the backhand of player two. This could be done performing exercises were the player has to hit at different part of the courts, dividing it in areas in a longitudinal direction or practising strokes modifying some parameters that could affect the flight of the ball such as the hitting speed, heights of the ball over the net (ropes could be used) or the ball spin. A modification of the racket parameters could also be considered, such as the swing-weight or tension of the string since they influence the accuracy (Allen et al., 2016; Bower \& Cross, 2005). Player one could be advised to work his backhand both laterally and longitudinally (his ellipse is less eccentric, has a shape more similar to a circle). It is also recommended that both perform exercises in which they are forced to target the baseline (the centre of their ellipses on the y-axis was positive, indicating that they had a tendency to perform short shots).

The present study has some limitations. Excel spreadsheet does not yet provide a database with which the user can compare the results of the players evaluated depending on the level of play, the age, the sex or the direction of the strokes. We believe that it is necessary to generate a database large enough to be able to evaluate the test results of a particular player, taking into account those characteristics. It would be interesting to translate the numerical results into more understandable adjectives for the coaches and to even classify the player at a game level based on the test results. Another possible limitation of this tool is that it is not automatic like other systems mentioned in the introduction. Although it requires a digitalization process, it presents certain strengths: it can be used with any conventional camera in complicated lighting conditions such as indoor tennis courts with low light or outdoor courts with contrast of light and shadow. Definitely we think that this research shows an interesting tool to investigate accuracy in detail in the case of tennis. Currently, the number of studies on accuracy and its relation with performance in tennis is rather scarce. Accuracy has been studied more in depth for other throwing sports such as baseball, cricket or handball (Freeston, Ferdinands, \& Rooney, 2015, 2007; Freeston \& Rooney, 2014; van den Tillaar \& Ettema, 2006). The test proposed in this work and the Excel tool that accompanies it is expected to be valuable for scientists who want to study accuracy. In our opinion, there are still a large number of factors that can affect accuracy during play and their impact should be studied in greater depth than what has been done so far, such as: fatigue (Lyons et al., 2013), racket characteristics (Bower \& Cross, 2005), mental aspects (Robin et al., 2007), variables related to the ball that approaches the player (Bower \& Sinclair, 2007) or with the ball that leaves the racket (Knudson \& Blackwell, 2005). Since the test does not require complex technical knowledge or setting-up, it is also expected that coaches with limited access to high end technologies can use it. In fact, the test could be applied at different times of the season to see progression of players in relation to their accuracy. This could be of special interest in high level players and professionals where changes may be less noticeable than in lower level players and where an area-based target system may not be sensitive 
enough to detect improvements in accuracy. In many cases, in addition to measuring the accuracy, it will be of interest to evaluate the speed of the stroke, since both variables are closely related to each other (Holzer, Reischl, \& Fetz, 1994 and Landlinger et al., 2012). The latter can be done with the use of a speed radar that measures the speed of the ball or with an inertial sensor that measures the speed of the racket.

\section{Conclusions}

The field test shown and the accompanying Excel spreadsheet provide valuable information for coaches and scientists and assist them in assessing the hitting accuracy with low cost and high precision. This paper shows an example of its implementation with two advanced level players. It was demonstrated how the outcomes of an affordable field test can easily be turned into tangible parameters that can inform training targets related to stroke accuracy, something which to our knowledge was previously not easy to perform.

\section{Disclosure statement}

No potential conflict of interest was reported by the authors.

\section{Acknowledgements}

The authors would like to thank the two athletes who participated in this research. It was supported by the Spanish Ministry of Education (FPU15/02949).

\section{References}

Allen, T., Choppin, S., \& Knudson, D. (2016). A review of tennis racket performance parameters. Sports Engineering, 19(1), 1-11. https://doi.org/10.1007/s12283-014-0167-x

Baiget, E., Fernández-Fernández, J., Iglesias, X., Vallejo, L., \& Rodríguez, F. A. (2014). On-Court Endurance and Performance Testing in Competitive Male Tennis Players. Journal of Strength and Conditioning Research, 28(1), 256-264. https://doi.org/10.1519/JSC.0b013e3182955dad
Bower, R., \& Sinclair, P. (2007). Tennis racket stiffness, string tension and impact velocity effects on post-impact ball angular velocity. Sports Engineering, 10, 111-122.

Bower, R., \& Cross, R. (2005). String tension effects on tennis ball rebound speed and accuracy during playing conditions. Journal of Sports Sciences, 23(7),

765-771. https://doi.org/10.1080/02640410400021914

Calvin, W. (1983). A stone's throw and its launch window: Timing precision and its implications for language and hominid brains. Journal of Theoretical Biology, 104, 121-135.

Chavda, S., Bromley, T., Jarvis, P., Williams, S., Bishop, C., Turner, A., Lake, J., \& Mundy, P. (2018). Force-time characteristics of the countermovement jump: Analyzing the curve in excel. Strength and Conditioning Journal, 40(2), 67-77. https://doi.org/10.1519/SSC.0000000000000353

Davey, P., Thorpe, R., \& Williams, C. (2003). Simulated tennis matchplay in a controlled environment. Journal of Sports Sciences, $21(6), 459-$ 467.

https://doi.org/10.1080/0264041031000101926

Delgado-García, G., Vanrenterghem, J., MuñozGarcía, A., Molina-Molina, A., \& Soto-hermoso, V. M. (2018). Does stroke performance in amateur tennis players depend on functional power generating capacity? The Journal of Sports Medicine and Physical Fitness. 59(5), 760-766. https://doi.org/10. 23736/S0022-4707.18.08518-3

Delgado-García, G., Vanrenterghem, J., MuñozGarcía, A., Ruiz-Malagón, E. J., Mañas-Bastidas, A., \& Soto-Hermoso, V. M. (2019). Probabilistic structure of errors in forehand and backhand groundstrokes of advanced tennis players. International Journal of Performance Analysis in Sport, 19(5), 698-710.. https://doi.org/10.1080/24748668. 2019.1647733

Dunn, M., Wheat, J., Miller, S., Haake, S., Goodwill, S., \& Federation, I. T. (2012). Reconstructing 2D Planar Coordinates Using Linear and Non- Linear Techniques. 30th Annual Conference on Biomechanics in Sports, (100), 380-383. 
Engström, E., Ottosson, E., Wohlfart, B., Grundström, N., \& Wisén, A. (2012). Comparison of heart rate measured by Polar RS400 and ECG, validity and repeatability. Advances in Physiotherapy, 14(3), 115-122.

Freeston, J., Ferdinands, R., \& Rooney, K. (2015). The Launch Window Hypothesis and the SpeedAccuracy Trade-Off in Baseball Throwing. Perceptual and Motor Skills, 121 (1), 135-148.

Freeston, J., Ferdinands, R., \& Rooney, K. (2007). Throwing velocity and accuracy in elite and sub-elite cricket players: A descriptive study. European Journal of Sport Science, 7(4), 231-237.

Freeston, J., \& Rooney, K. (2014). Throwing Speed and Accuracy in Baseball and Cricket Players. Perceptual and Motor Skills, 118(3), 637-650. https://doi.org/10.2466/30.PMS.118k25w4

Holzer, E., Reischl, G., \& Fetz, F. (1994). Genauigkeitstraining bei Ballsportarten. ÖBV-Pädagog. Verlag.

International Tennis Federation (ITF). About International Tennis number. Retrieved from http://www.tennisplayandstay.com/itn/about-theitn/about-the-itn.aspx (accessed 7 July 2019).

Knudson, D. , \& Blackwell, J. (2005). Variability of impact kinematics and margin for error in the tennis forehand of advanced players. Sports Engineering, 8(2), 75-80. https://doi.org/10.1007/BF02844005

Landlinger, J., Stöggl, T., Lindinger, S., Wagner, H., \& Müller, E. (2012). Differences in ball speed and accuracy of tennis groundstrokes between elite and high-performance players. European Journal of Sport Science, $12(4)$, 301-308. https://doi.org/10.1080/17461391.2011.566363

Lyons, M., Al-Nakeeb, Y., Hankey, J., \& Nevill, A. (2013). The effect of moderate and high-intensity fatigue on groundstroke accuracy in expert and nonexpert tennis players. Journal of Sports Science and Medicine, 12(2), 298-308.

Messelodi, S., Modena, C. M., Ropele, V., Marcon, S., \& Sgrò, M. (2019). A Low-Cost Computer Vision System for Real-Time Tennis Analysis. International Conference on Image Analysis and Processing, 106-116. Springer.
Reid, M., Morgan, S., \& Whiteside, D. (2016). Matchplay characteristics of Grand Slam tennis: implications for training and conditioning. Journal of Sports Sciences, 34(19), 1791-1798. https://doi.org/10.1080/02640414.2016.1139161

Robin, N., Dominique, L., Toussaint, L., Blandin, Y., Guillot, A., \& Her, M. (2007). Effects of motor imagery training on service return accuracy in tennis: The role of imagery ability. International Journal of Sport and Exercise Psychology, 5(2), 175-186. https://doi.org/10.1080/1612197x.2007.9671818

Schubert, P., \& Kirchner, M. (2014). Ellipse area calculations and their applicability in posturography. Gait and Posture, 39(1), 518-522. https://doi.org/10.1016/j.gaitpost.2013.09.001

Shinya, M., Tsuchiya, S., Yamada, Y., Nakazawa, K., Kudo, K., \& Oda, S. (2017). Pitching form determines probabilistic structure of errors in pitch location. Journal of Sports Sciences, 35(21), 2142-2147. https://doi.org/10.1080/02640414.2016.1258484

Smekal, G., Pokan, R., Von Duvillard, S. P., Baron, R., Tschan, H., \& Bachl, N. (2000). Comparison of laboratory and on-court endurance testing in tennis. International Journal of Sports Medicine, 21 (04), 242249.

Strecker, E., Foster, E., \& Pascoe, D. (2011). Testretest Reliability for Hitting Accuracy Tennis Test. Journal of Strength and Conditioning Research, 25(12), 3501-3505.

https://doi.org/10.1519/JSC.0b013e318215fde6

van den Tillaar, R., \& Ettema, G. (2006). A Comparison between Novices and Experts of the Velocity-Accuracy Trade-Off in Overarm Throwing. Perceptual and Motor Skills, 103(2), 503-514. https://doi.org/10.2466/pms.103.2.503-514

Vergauwen, L., Brouns, F., \& Hespel, P. (1998). Carbohydrate supplementation to improve stroke performance in tennis. Medicine $\mathcal{E}$ Science in Sports $\mathcal{E}$ Exercise, 30(8), 1289-1295.

Vergauwen, L., Spaepen, A., Lefevre, J., \& Hespel, P. (1998). Evaluation of stroke performance in tennis. Medicine and Science in Sports and Exercise, 30(8), 1281-1288.

Wawrzyniak, Z. M., \& Kowalski, A. (2016). Eventbased image recognition applied in tennis training 
assistance. Photonics Applications in Astronomy, Communications, Industry, and High-Energy Physics Experiments 2016, 10031, 100311N. International Society for Optics and Photonics.

Wei, X., Lucey, P., Morgan, S., \& Sridharan, S. (2013). Sweet-Spot: Using Spatiotemporal Data to Discover and Predict Shots in Tennis. Retrieved from https://pdfs.semanticscholar.org/cf6c/b34cdc0c2ed d03cd2e6217c8b94b6acd506c.pdf
Yamamoto, H., Shinya, M., \& Kudo, K. (2018). Cognitive Bias for the Distribution of Ball Landing Positions in Amateur Tennis Players (Cognitive Bias for the Motor Variance in Tennis). Journal of Motor Behavior, 2895 , $1-10$. https://doi.org/10.1080/00222895.2018.1440523

Zaiontz C. (2018) Real Statistics Using Excel. Retrieved from www.real-statistics.com. 